\title{
CT45A1 acts as a new proto-oncogene to trigger tumorigenesis and cancer metastasis
}

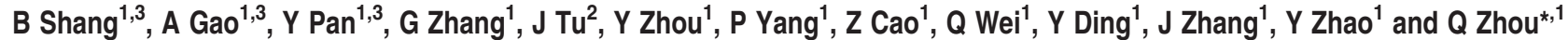

Cancer/testis antigen (CTA)-45 family (CT45) belongs to a new family of genes in phylogenetics and is absent in normal tissues except for testis, but is aberrantly overexpressed in various cancer types. Whether CT45 and other CTAs act as proto-oncogenes has not been determined. Using breast cancer as a model, we found that CT45A1, a representative CT45 family member, alone had a weak tumorigenic effect. However, its neoplastic potency was greatly enhanced in the presence of growth factors. Overexpression of CT45A1 in breast cancer cells markedly upregulated various oncogenic and metastatic genes, constitutively activated ERK and CREB signaling pathways, promoted epithelial-mesenchymal transition, and increased cell stemness, tumorigenesis, invasion, and metastasis, whereas silencing CT45A1 significantly reduced cancer cell migration and invasion. We propose that CT45A1 functions as a novel proto-oncogene to trigger oncogenesis and metastasis. CT45A1 and other CT45 members are therefore excellent targets for anticancer drug discovery and targeted tumor therapy, and valuable genes in the study of a molecular phylogenetic tree.

Cell Death and Disease (2014) 5, e1285; doi:10.1038/cddis.2014.244; published online 5 June 2014

Subject Category: Cancer

\section{Introduction}

Carcinogenesis is a dynamic process dependent on not only the oncogenic properties of cancer cells but also dependent on a favored environment in organs. In particular, genetic and epigenetic abnormalities arise in a hostile tumor microenvironment, leading to the activation of various proto-oncogenes, to produce numerous oncogenes, resulting in tumor initiation, progression, and metastasis. ${ }^{1}$ In the search for cancerassociated genes, various cancer/testis (CT) antigens (CTAs) have been found to be aberrantly overexpressed in cancer types. $^{2,3}$ CTAs originally referred to testis-derived specific immunogenic antigens that elicited spontaneous immune responses in cancer patients. ${ }^{4-6}$ They are not expressed in nearly all tissues after birth, except for the testis, ${ }^{3}$ but are highly expressed in cancer types, ${ }^{2,3,6-14}$ and are closely associated with poor overall survival of cancer patients. ${ }^{7-14}$ Presently, more than 200 CT genes have been identified. ${ }^{15}$ However, whether CTAs exert an oncogene-like function is still unknown. ${ }^{2,3}$

Among CTAs, the CT45 gene family (CT45) is especially important because of its unique genetic characteristics and aberrant expression in various cancer types. CT45 comprises six genes designated as CT45A1 to CT45A6, which are clustered in tandem within a 125-kb-narrow region at chromosome Xq26.3 ${ }^{15,16}$ (Supplementary Figure S1a). The amino-acid sequences exhibit more than $98 \%$ identity among the six CT45 family members (Supplementary Figure S1b). CT45 exists only in Homo sapiens and 10 other primates, and not in any other species, and belongs to a new gene family in terms of biological evolution ${ }^{15}$ (Supplementary Figure S1c). In normal human, CT45 is only expressed in the testis, and not in any other tissues, but is overexpressed in various malignant tumors, including lung cancer, ${ }^{16}$ breast cancer, ${ }^{16-18}$ and so on. ${ }^{19,20}$ Notably, overexpression of CT45 is closely associated with tumor progression, aggressiveness, and poor prognosis. ${ }^{17-23}$ Despite the close association of CT45 overexpression with poor prognosis of cancer patients, the biological function of CT45 is still less studied. Presently, only Koop et al. ${ }^{24}$ recently reported that downregulation of CT45 expression in lymphoma, fibrosarcoma, and myeloma cell lines, using siRNA, changed tumor cell morphology and reduced cell migration; however, the possible role of CT45 in carcinogenesis is still unclear.

In addition, CT45 was aberrantly overexpressed in nonHodgkin's lymphoma (NHL) patients, and very high titers against CT45 in two diffuse large B-cell lymphoma patients were found in 97 untreated $\mathrm{NHL}$ serum samples, which conceptually demonstrated that CT45 was able to elicit

\footnotetext{
${ }^{1}$ Cyrus Tang Hematology Center, Jiangsu Institute of Hematology, The First Affiliated Hospital of Soochow University, Key Laboratory of Thrombosis and Hemostasis, Ministry of Health, Soochow University, Suzhou, Jiangsu, China and ${ }^{2}$ The Second Affiliated Hospital of Soochow University, Soochow University, Suzhou, Jiangsu, China

${ }^{*}$ Corresponding author: Q Zhou, Cyrus Tang Hematology Center, Jiangsu Institute of Hematology, The First Affiliated Hospital of Soochow University, Soochow University, 199 Ren Ai Road, Suzhou Industrial Park, Suzhou 215123, China. Tel: + 86 (512) 65882116; Fax: +86 (512) 65880929; E-mail: quanshengzhou@yahoo.com

${ }^{3}$ These authors contributed equally to this work.

Abbreviations: CTAs, Cancer/testis(CT) antigens; CT45, CT45 gene family; CT45A1, cancer/testis antigen-45A1; EMT, epithelial-mesenchymal transition; EGF, epithelial growth factor; bFGF, basic fibroblast growth factor; SF-EFB, serum-free DMEM/F12 medium supplemented with $10 \mathrm{ng} / \mathrm{ml}$ EGF, $10 \mathrm{ng} / \mathrm{ml}$ bFGF, and $1 \times$ B27; ALDH1A1, aldehyde dehydrogenases 1 family, member A1; SULF2, sulfatase 2; MAGED4B, melanoma antigen family D-4B; HOXB6, homeobox B6; RASEGF1A, RAS exchange factor A1; UV, ultraviolet; H\&E, hematoxylin and eosin; WB, western blotting; IF, immunofluorescence; PI, propidium iodide; DAPI, 4.6-diamidino-2phenylindole; ECL, chemiluminescence
}

Received 13.2.14; revised 25.4.14; accepted 29.4.14; Edited by A Stephanou 
spontaneous humoral immune response in some $\mathrm{NHL}$ patients, ${ }^{14}$ warranting for further investigation of the immunogenic properties of the CT45 family in various other cancer types and CT45 as potential targets for cancer immunotherapy.

Breast cancer is a leading malignant tumor in females. Several proto-oncogenes are highly activated during the development of this malignancy. ${ }^{25}$ Whether CT45 functions as a proto-oncogene to drive tumorigenesis has not been determined. CT45 genes are associated with poor prognosis of cancer patients: ${ }^{16-23}$ we hypothesize that CT45 may act as a proto-oncogene to trigger oncogenesis.

In the current study, we used low tumorigenic breast cancer MCF7 cells, which lack CT45A1 and other CT45 family members, as a model to explore the oncogenic effect of the gene and its mechanisms, and we found that overexpression of CT45A1 notably increased tumorigenesis, invasion, and metastasis of breast cancer cells in a growth factor-dependent manner. Mechanistic studies revealed that CT45A1 markedly upregulated several key tumorigenic and metastatic genes, induced epithelial-mesenchymal transition (EMT), increased cell stemness, and enhanced breast cancer cell invasion and metastasis. These results suggest that CT45A1 acts as a newly discovered proto-oncogene, which can trigger tumorigenesis and cancer metastasis in a favored tumor microenvironment.

\section{Results}

CT45A1 acts as a new proto-oncogene to trigger tumorigenesis and metastasis of breast cancer. Taking advantage of the breast cancer cell line MCF7, which does not express the six CT45 genes and possesses very low tumorigenicity in vitro and in vivo, we selected CT45A1 as a representative of the CT45 family. Initially, we established CT45A1-positive and -negative MCF7 cell lines by infection of cells with lentivirus, containing either Venes-CT45A1-IRES-GFP or Venes-IRES-GFP, respectively. GFP-positive cells were sorted with flow cytometry (Supplementary Figure S2a), and expression of CT45A1 in the infected MCF7 cells was confirmed with RT-PCR (Supplementary Figure S2b), western blotting (WB) analysis (Supplementary Figure S2c), and immunofluorescent staining (Supplementary Figure S2d). CT45A1-infected MCF7 cells were named MCF7-CT45A1, and the vector mock-infected MCF7 cells were named MCF7-V.

When MCF7-CT45A1 or MCF7-V cells were cultured in routine cell culture complete medium containing $10 \%$ fetal bovine serum (complete medium), some of the MCF7CT45A1 cells displayed morphological alterations. The cell nucleus was enlarged, whereas the cell cytosol was reduced; however, there were no obvious morphological changes in the control MCF7-V cells (Figure 1a). When the cells were cultured for 5 days in a serum-free medium supplemented with SF-EFB consisting epithelial growth factor (EGF), basic fibroblast growth factor (bFGF), and B27 neuron survival factor, the majority of MCF7-V cells still adhered to the bottom of the flask, and only a minor portion of the cells formed small and adhesive mammospheres (Figure 1b, left panel). By contrast, MCF7-CT45A1 cells formed larger mammospheres, and most of the mammospheres were suspended in cell culture medium (Figure $1 \mathrm{~b}$, right panel). These results suggested that the overexpression of CT45A1 can induce MCF7 cell dedifferentiation and EMT.

Next, we investigated whether the overexpression of CT45A1 also promotes tumorigenesis of MCF7 cells using a colony-forming assay in the absence of growth factors. When both cell types were cultured in complete medium, MCF7-CT45A1 cells formed more colonies than the control MCF7-V cells, and the colony sizes of MCF7-CT45A1 cells were larger than the colonies from control MCF7-V cells (Figures 1c and d). After the cells were cultured in SF-EFB for 5 days, both the colony number and size were markedly increased in MCF7-CT45A1 cells when compared with the control cells (Figures $1 e$ and $\mathrm{f}$ ). To our surprise, overexpression of CT45A1 did not affect cell growth rate (Supplementary Figure S3a) and cell cycle parameters (Supplementary Figure S3b). In addition, MCF7-CT45A1 cells had diminished apoptosis when they were ultraviolet (UV)-irradiated, as compared with control MCF7-V cells (Supplementary Figure S4a-c). Together, these in vitro data suggest that CT45A1 promotes MCF7 cell dedifferentiation and EMT, and increases cell stemness and tumorigenesis in a growth factor-dependent manner.

Next, we characterized the in vivo tumorigenesis of CT45A1. Using xenografted mice, in situ breast tissue received either MCF7-CT45A1 or control MCF7-V cells without administration of estrogen. Tumors in the MCF7-CT45A1 group grew faster than the control MCF7-V group (Figure 2a), and the tumor size was larger and heavier in the MCF7-CT45A1 group compared with the control group (Figures $2 b$ and $c$ ). Notably, in the MCF7-CT45A1xenografted mice, the tumor cells invaded the adjacent tissue of the rib bone (Figure 2d, right), whereas the tumor in the control MCF7-V mice was well isolated in the breast tissue without invasion of neighboring tissue (Figure 2d, left). Hematoxylin and eosin (H\&E) staining of the lung tissue showed multiple metastatic sites in several xenografted MCF7-CT45A1 mice, with the absence of any lung metastasis in all of the MCF7-V-xenografted mice (Figure $2 \mathrm{e}$ ). In addition, immunofluorescent staining indicated that both CT45A1 and CD44 were present in lung metastatic tumors of MCF7CT45A1-xenografted mice, but were absent in lung tissue of the control MCF7-V-xenografted mice (Figure 2f).

To further study the role of CT45A1 in tumor cell invasion and metastasis, we performed MCF7 cell migration and invasion assays in vitro. The wound-healing assay indicated that the gap in the MCF7-CT45A1 cell monolayer was more rapidly closed over $48 \mathrm{~h}$ as compared with that of control MCF7-V cells (Figures $3 a$ and $b$ ). In addition, overexpression of CT45A1 significantly increased the invasion of MCF7CT45A1 cells compared with control MCF7-V cells (Figures $3 c$ and d).

Besides, we examined CT45A1-mediated cell morphological and functional changes in other two lines, which do not express CT45A1, one is breast cancer cell line Hs578T and the other is gastric cancer cell line AGS. CT45A1-negative Hs578T and AGS cells were infected with the lentivirus containing either Venes-CT45A1-IRES-GFP or control Venes-IRES-GFP, respectively; GFP-positive cells were 


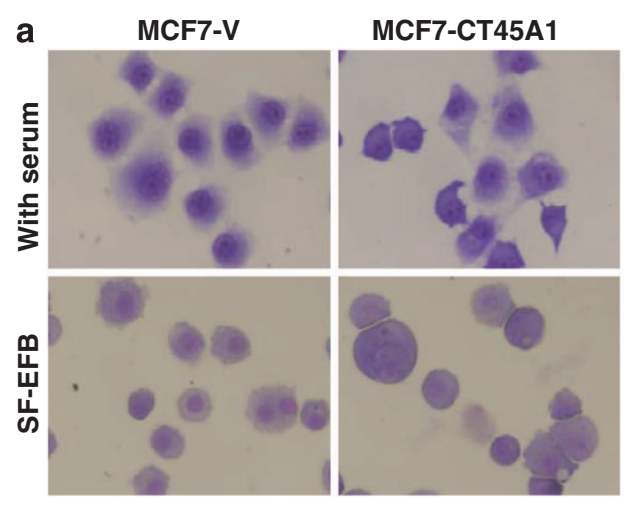

C

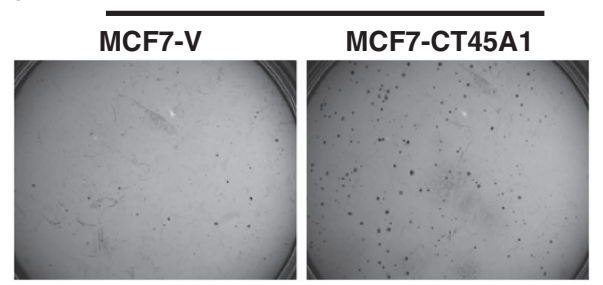

d

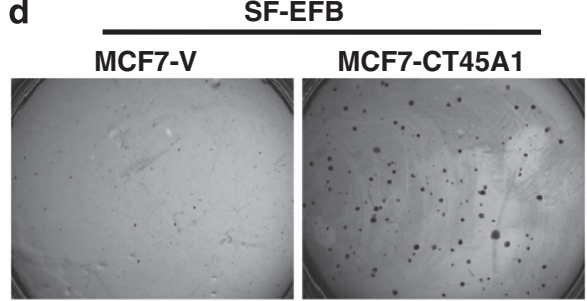

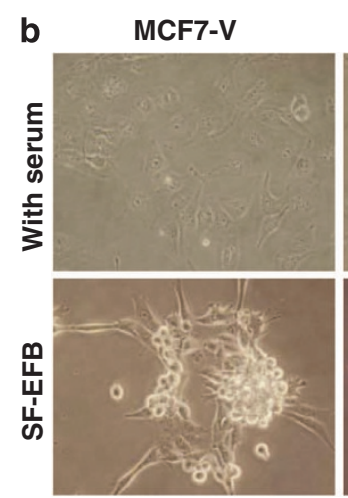

MCF7-CT45A1
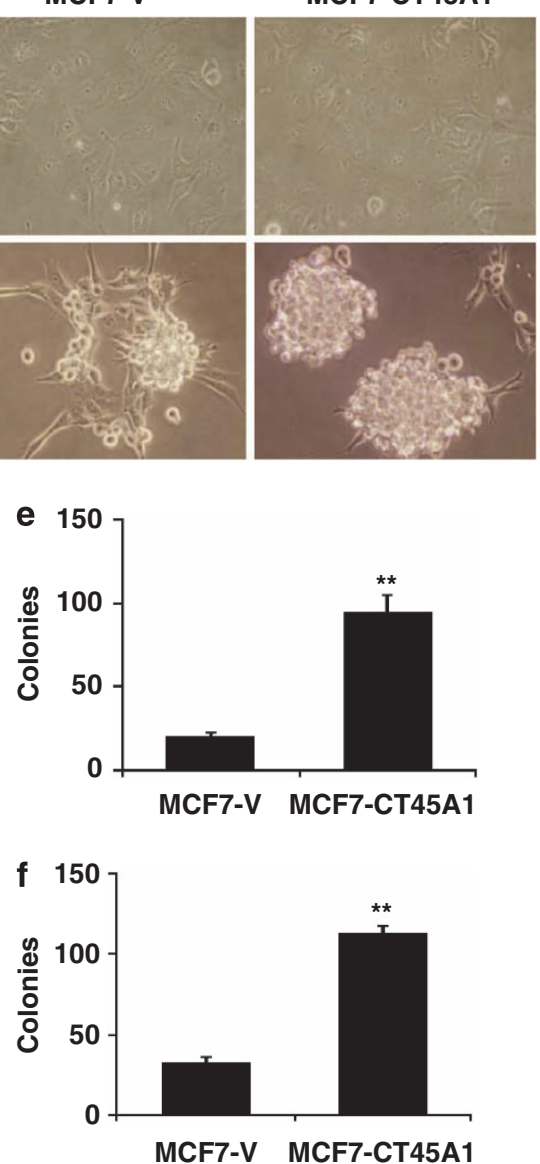

Figure 1 CT45A1 induces breast cancer cell EMT and promotes tumorigenesis. After MCF7-V and MCF7-CT45A1 cells were cultured in either the complete medium (with serum) or serum-free medium (SF-EFB), the cells were stained with Wright-Giemsa solution and photographed with amplification of $\times 200$ (a), the formation of mammospheres was directly imaged at $\times 200$ (b), the colonies were photographed, $\times 5$ (c and e), and counted under the microscopy (d and f). The data were a representative of three repeats. Samples were compared using one-way ANOVA. Error bars indicated mean \pm S.D., ${ }^{* *} P<0.01$, as compared with MCF7-V cells

sorted using flow cytometry (Supplementary Figure S5a), and the expression of CT45A1 in the infected Hs578T and AGS cells was confirmed using western blot analysis (Supplementary Figure S5b). The CT45A1-positive Hs578T and AGS cells were named Hs578T-CT45A1 and AGSCT45A1, and the vector mock-infected CT45A1-negative cells were called Hs578T-V and AGS-V, respectively. WrightGiemsa staining showed that some of the Hs578T-CT45A1 cells displayed morphological alterations compared with the control Hs578T-V cells; in a portion of CT45A1-infected cells, the cell cytoplasm was reduced and the nucleus was deeply stained (Supplementary Figure S5c), the result is consistent with the morphological changes in the MCF7 cells overexpressing CT45A1 mentioned above (Figure 1a).

Next, we investigated whether overexpression of CT45A1 changes cell function as we observed in MCF7 cells. Overexpression of CT45A1 in Hs578T and AGS cells did not have an effect on cell growth and cell cycles (data not shown); however, the migration of CT45A1-positive Hs578T-CT45A1 and AGS-CT45A1 cells was significantly faster than the control CT45A1-negative Hs578T-V and AGS-V cells (Supplementary Figures $\mathrm{S} 6 \mathrm{a}$ and b). In addition, overexpression of CT45A1 significantly enhanced the invasion of
Hs578T-CT45A1 and AGS-CT45A1 cells (Supplementary Figures $\mathrm{S7a}$ and $\mathrm{b}$ ). In short, these data are compatible with the function of CT45A1 in breast cancer MCF7 cells as stated before, suggesting that $\mathrm{CT} 45 \mathrm{~A} 1$ promotes tumor cell migration and invasion.

Furthermore, to confirm the pro-metastatic effects of CT45A1, we used shRNA to knockdown CT45A1 in the metastatic gastric cancer cell line SGC7901, which constitutively overexpressed CT45A1. Four CT45A1 shRNAs were synthesized and cloned into lentivirus-IRES-GFP vector and used to transfect package cell 293T to produce infectious lentivirus. SGC7901 cells were infected with lentivirus containing one of the four CT45A1 shRNAs, either shRNA19747, shRNA19748, shRNA19749, or shRNA1, respectively. GFP-positive infected cells were sorted (Supplementary Figure S8a). CT45A1 expression levels in the cells were examined using western blot analysis (Supplementary Figure S8b) and immunofluorescent staining (Supplementary Figure S8c). WB and immunofluorescent staining showed that CT45A1-shRNA1 effectively silenced CT45A1 expression in SGC7901 cells, and the level of CT45A1 was reduced more than $95 \%$ in the cells, whereas CT45A1-shRNA19749 did not affect CT45A1 level in the 

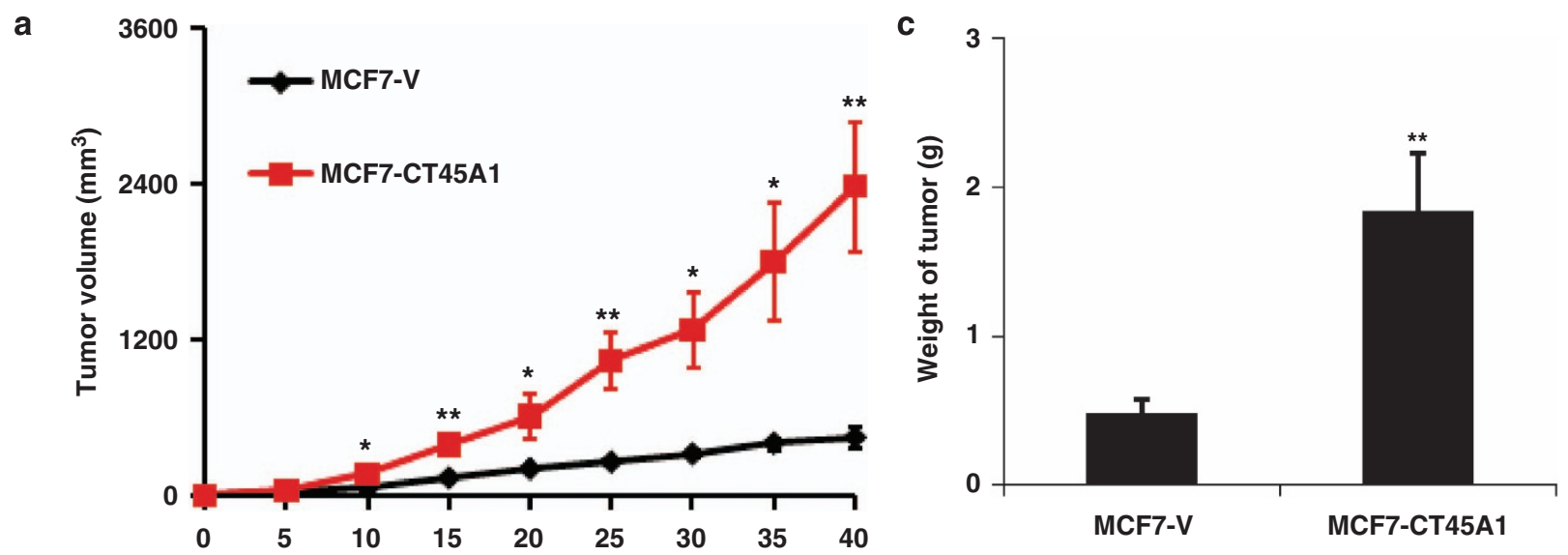

b

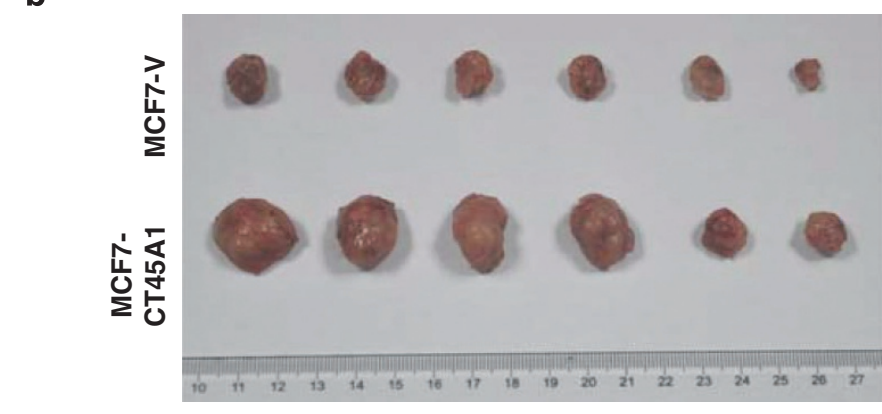

d

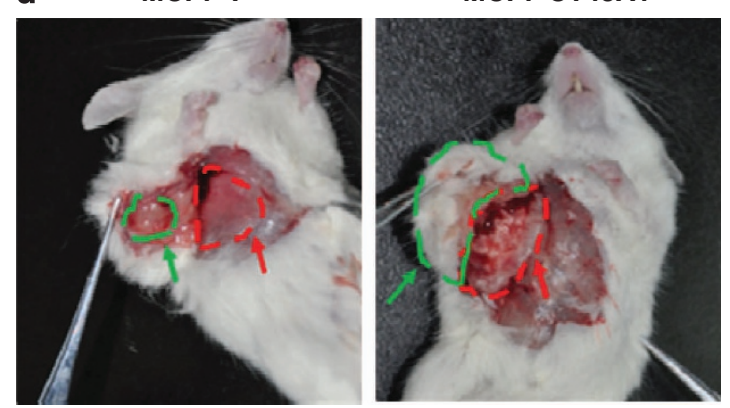

MCF7-CT45A1
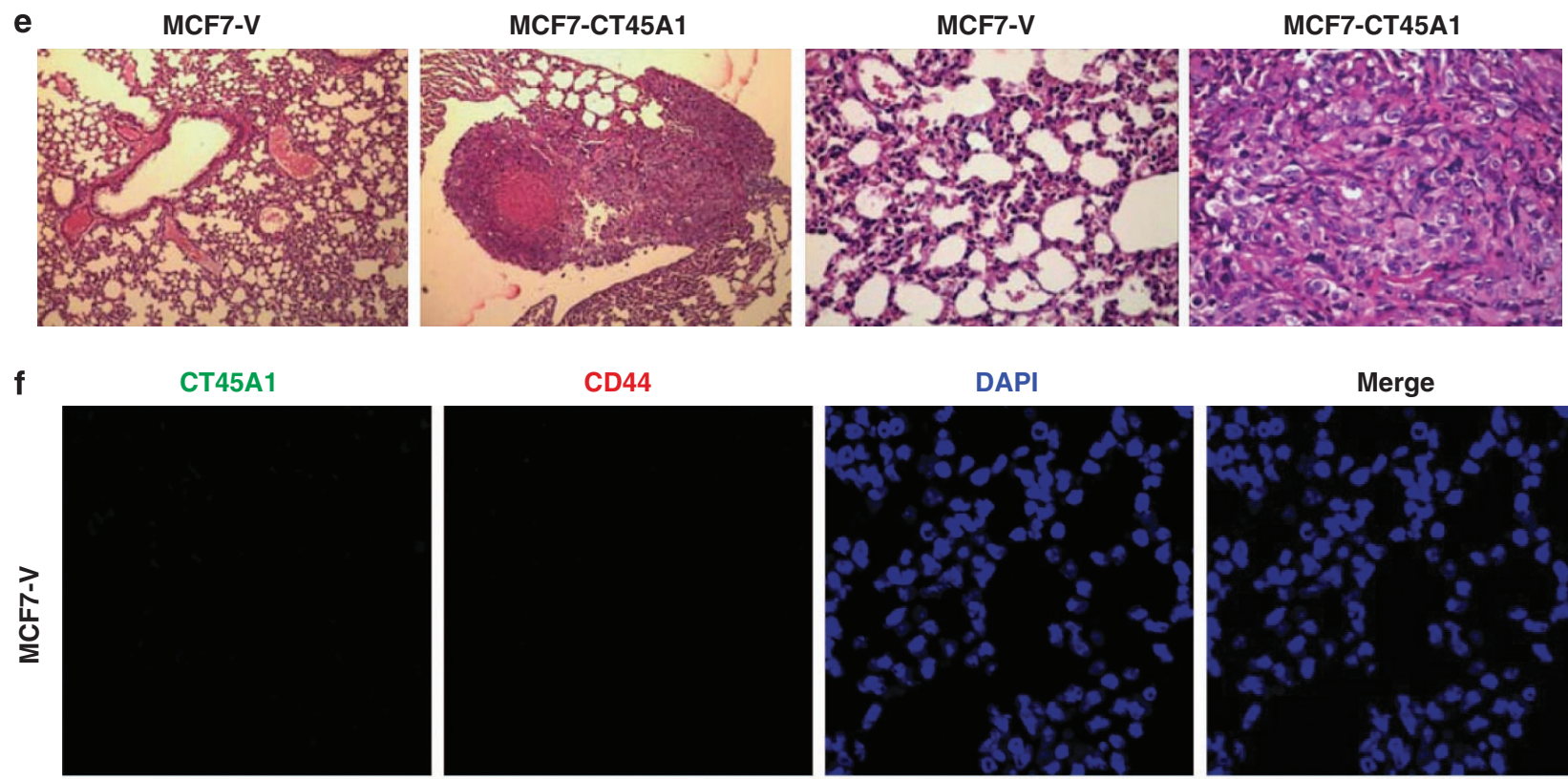

CD44

DAPI

Merge
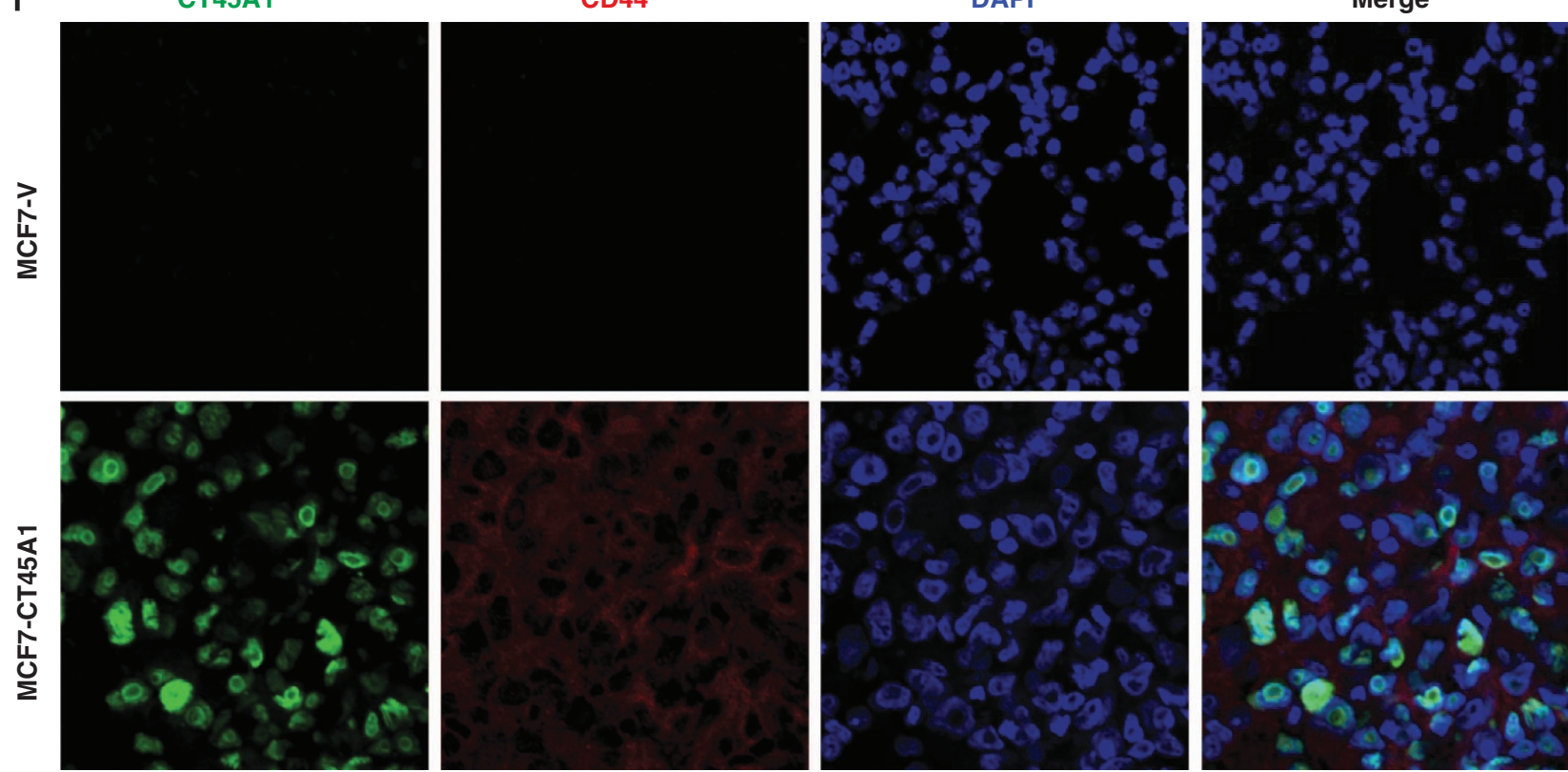
SGC7901 cells (Supplementary Figures S8b and c). Accordingly, we chose CT45A1-positive (SGC7901-shRNA19749) and -silenced (SGC7901-shRNA1) SGC7901 cells as models to access the function of CT45A1. Wright-Giemsa staining displayed that cell morphology altered in most CT45A1silenced SGC7901-shRNA1 cells compared with the CT45A1-positive SGC7901-19749 cells; obviously, cell size was enlarged and the cell cytosol was also increased in CT45A1-silenced SGC7901-shRNA1 cells (Figure 4a). Cell migration assay showed that knocking down CT45A1 expression significantly reduced the migration of SGC7901shRNA1 cells (Figure 4b). In addition, silencing CT45A1 also decreased the invasion of SGC7901-shRNA1 cells (Figure 4c). Collectively, these data imply that silencing CT45A1 in SGC7901 diminishes the migration and invasion of the metastatic gastric cancer cells.

The mechanisms of CT45A1-mediated tumorigenesis and cancer metastasis. To determine the mechanisms of CT45A1-mediated tumorigenesis and cancer metastasis, we used a DNA microarray to analyze the differential gene expression profile between MCF7-CT45A1 and MCF7-V cells. The results indicated that the overexpression of CT45A1 in MCF7 cells greatly changed the gene expression profile. With threefold or greater changes as a cutoff threshold, among the 32375 genes on the array, 950 genes were upregulated (Supplementary Table 1) and 1092 genes were downregulated (Supplementary Table 2). Importantly, several genes responsible for EMT, stemness, tumorigenesis, and metastasis were markedly upregulated in MCF7CT45A1 as compared with the control MCF7-V cells (Figures 5 and 6). In light of that definitive important tumor suppressor genes were not downregulated in the gene expression profiling, we focused on these CT45A1-upregulated genes in the current investigation. The DNA microarray results were further confirmed using RT-PCR and real-time quantitative PCR (QT-PCR). Overexpression of CT45A1 upregulated a master EMT gene TWIST1, whereas other EMT genes were unchanged (Figure 5a). QT-PCR showed that TWIST1 expression was increased 34.3-fold in MCF7-CT45A1 cells compared with control MCF7-V cells (Figure $5 \mathrm{~b}$ ). In addition, among the nine cancer stem cell-related genes examined, a
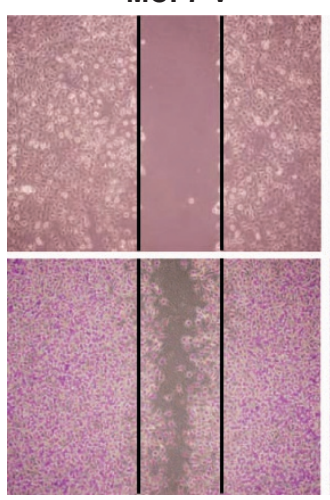

C

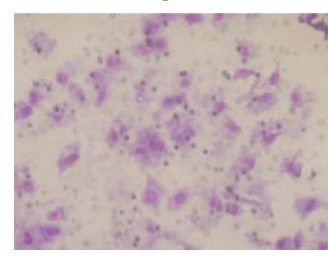

MCF7-CT45A1
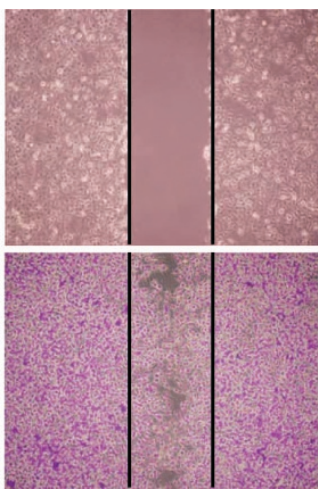

MCF7-CT45A1

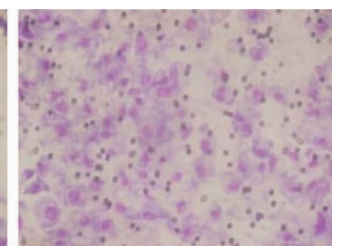

b
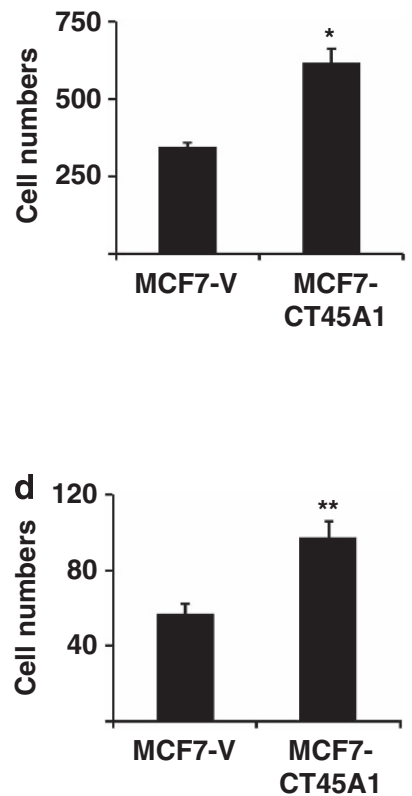

Figure 3 CT45A1 enhances cell migration and invasion. The migration of MCF7-V and MCF7-CT45A1 cells was measured with the wound-healing assay ( $n=3$ ). After culture for $48 \mathrm{~h}$, the cells were stained with Wright-Giemsa solution and photographed at $\times 40$ (a and $\mathbf{b}$ ); the invasion of the cells was measured using the transwell assay $(n=3)$. After culture for $24 \mathrm{~h}$, the cells were stained with Wright-Giemsa solution and photographed at $\times 200$ (c and d). Samples were compared using one-way ANOVA, and error bars represent mean \pm S.D., ${ }^{\star} P<0.05,{ }^{\star \star} P<0.01$, as compared with MCF7-V cells

Figure 2 CT45A1 enhances tumorigenesis in breast cancer in situ xenografted mice. After MCF7-V or MCF7-CT45A1 cells were cultured with SF-EFB for 5 days, the cells were harvested and injected into the breast cushion of NOD-SCID mice $(n=6)$, and the tumor volume was measured every other days (a), the excised tumors were photographed (b), and weighted (c). The tumor invasion in the breast area of the mice was photographed (d), the green line marked the breast in situ xenografted area, and the red line marked the rib bone area; tumor cell invasion to the vicinity of the rib bone was noted in MCF7-CT45A1 cell-xenografted mice (d, right panel), but absent in CT45A1negative MCF7-V cell-xenografted mice (d, left panel). The experiments were repeated twice. Samples were compared using one-way ANOVA, and error bars of the data indicated mean \pm S.E.M., ${ }^{*} P<0.05,{ }^{* *} P<0.01$, as compared with MCF7-V cells. The lung tissues in MCF7-V and MCF7-CT45A1 cell-xenografted mice were excised and sectioned. The tissue slides were stained with H\&E method and imaged at $\times 100$ (e, left two panels) and $\times 400$ (e, right two panels); (f) in addition, the slides were triply stained with CT45A1-specific antibody (green color), breast cancer cell marker CD44 antibody (red color), and DAPI (blue color), and photographed with confocal microscopy at $\times 1000$ 


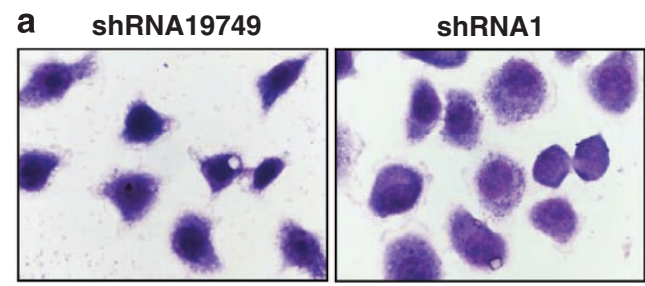

b

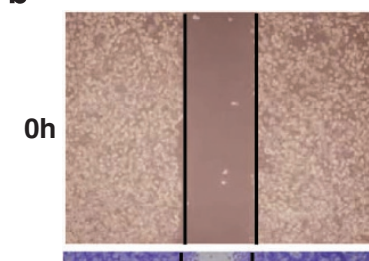

ShRNA19749

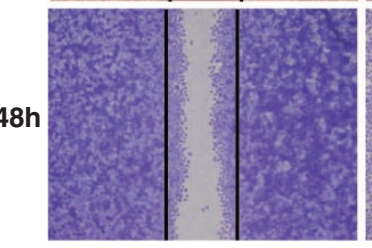

c

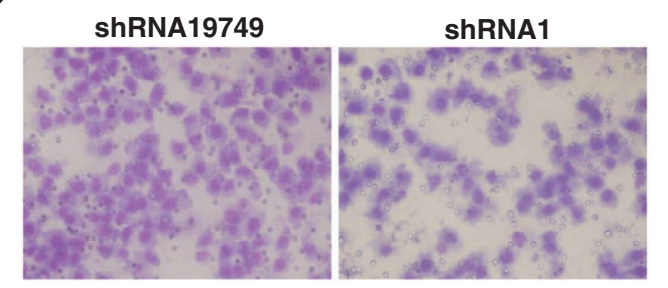

ShRNA1

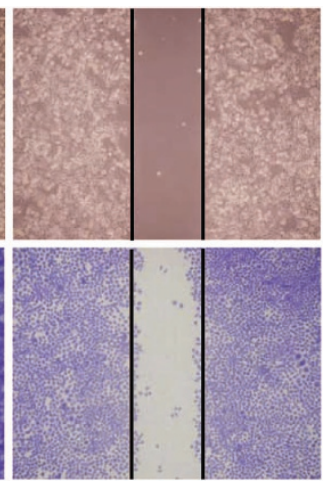

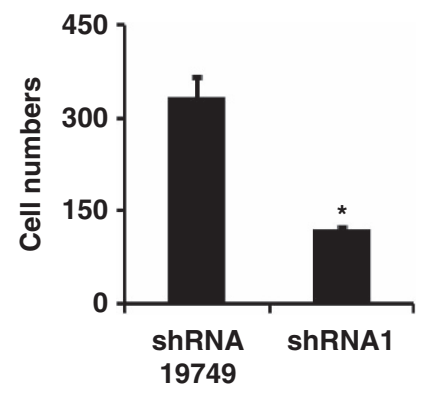

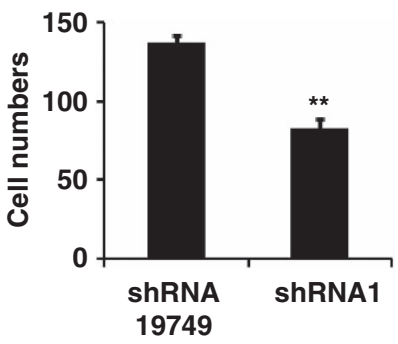

Figure 4 Silencing CT45A1 diminishes cancer cell migration and invasion. SGC7901-shRNA19749 and SGC7901-shRNA1 cells were stained with Wright-Giemsa solution and photographed at $\times 1000$ (a). The migration of the cells was measured using the wound-healing assay $(n=3)$. After culture for $48 \mathrm{~h}$, the cells were stained with Wright-Giemsa solution and photographed at $\times 40(\mathrm{~b})$. The invasion of the cells was measured using the transwell assay $(n=3)$. After culture for $24 \mathrm{~h}$, the cells were stained with Wright-Giemsa solution and photographed at $\times 200$ (c). The data were analyzed using one-way ANOVA, and error bars represent mean \pm S.D., ${ }^{*} P<0.05,{ }^{* \star} P<0.01$, as compared with SGC7901-shRNA19749 cells

expression of aldehyde dehydrogenases 1 family, member A1 (ALDH1A1), and KIT (CD117) was significantly higher in MCF7-CT45A1 cells than control MCF7-V cells, whereas the other seven stemness-related genes were unchanged (Figure 5a). QT-PCR showed that expression of ALDH1A1 and KIT were elevated 17.8- and 3.0-fold, respectively, in MCF7-CT45A1 cells in comparison with control MCF7-V cells (Figures $5 c$ and $d$ ).

Expression of several cancer metastatic genes was also upregulated in MCF7-CT45A1 cells (Figure 5a). Notably, expression of the sulfatase 2 (SULF2) gene was elevated 153.9-fold in MCF7-CT45A1 cells and expressions of the CXCR4 gene in MCF7-CT45A1 cells were increased 7.0-fold, compared with control cells (Figures $5 e$ and $5 f$ ). Collectively, these data suggest that CT45A1 activates overexpression of various oncogenic and metastatic genes, which have important roles in carcinogenesis and tumor dissemination.

In addition, RT-PCR showed that several oncogenic genes, including melanoma antigen family D-4B (MAGED4B), homeobox B6 (HOXB6), HOXD13, and RAS exchange factor A1 (RASEGF1A) were markedly overexpressed in
MCF7-CT45A1 cells compared with control MCF7-V cells (Figure 6a). QT-PCR showed that expression of MAGED4B, HOXB6, HOXD13, and RASEGF1A were increased 144.3-, 9.5-, 26.9-, and 3.42-fold, respectively, in MCF7-CT45A1 cells as compared with control MCF7-V cells (Figures $6 b-e)$.

We also found that phosphorylation of ERK and CREB was higher in MCF7-CT45A1 cells than control MCF7-V cells in the absence of growth factor stimulation, but total ERK and CREB protein levels were unchanged, suggesting that overexpression of CT45A1 constitutively increased ERK and CREB signaling pathways (Figures $7 \mathrm{a}-\mathrm{C}$ ). In addition, phosphorylation of AKT was significantly enhanced in the presence of growth factors (Figures 7a and d); however, phosphorylation of several other signaling proteins, such as STAT3, JNK, and $\mathrm{NF}-\kappa \mathrm{B}$, was unaffected (Figure 7a). Immunofluorescent staining and confocal microscopy showed that overexpression of CT45A1 in MCF7 resulted in the localization of phosphorylated ERK in the cell nucleus (Figure 7e). CT45A1 was also colocalized in the cell nucleus, suggesting that CT45A1 may have an important role in oncogenic signaling pathways. 


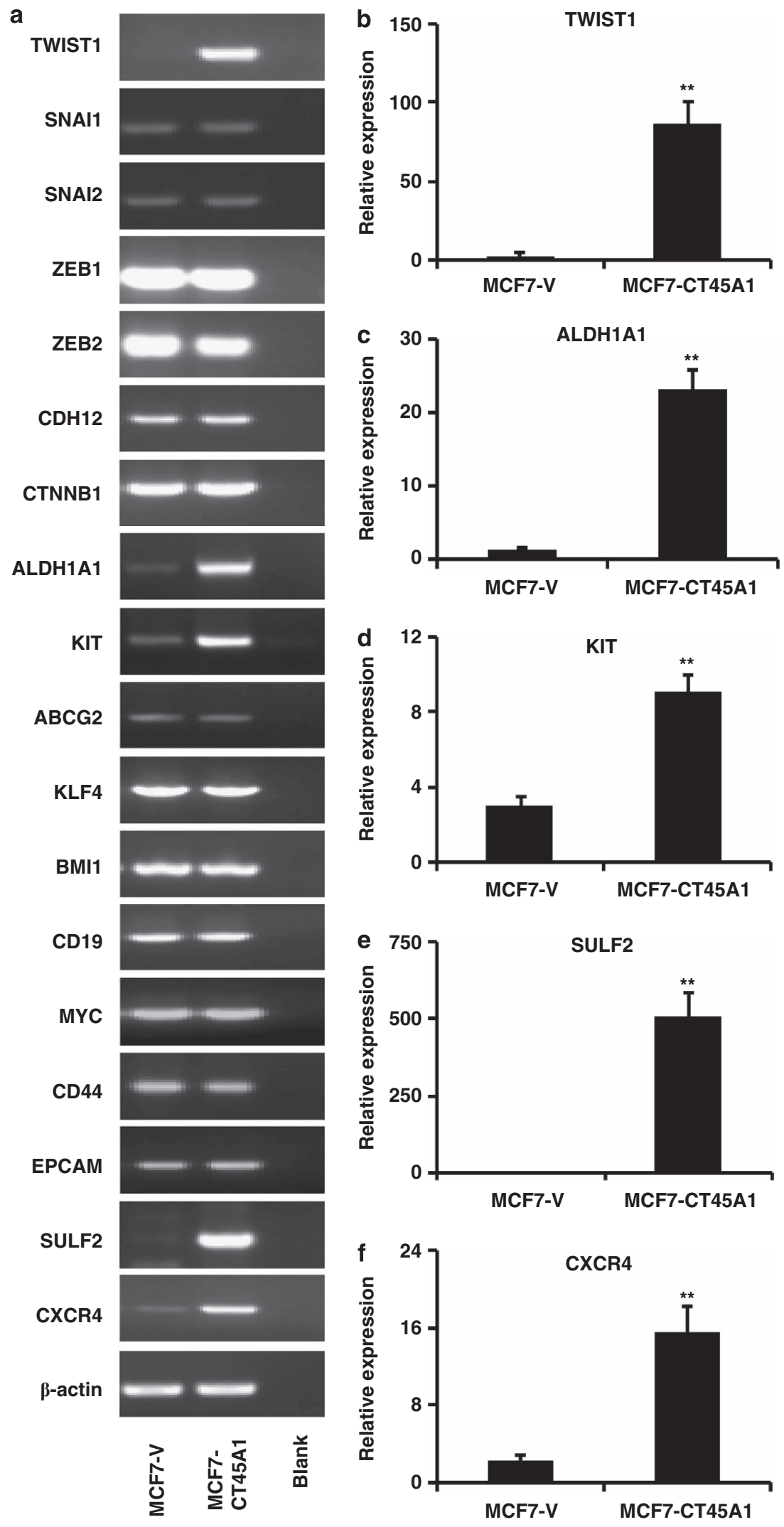

Figure 5 CT45A1 induces overexpression of EMT, stemness, and metastatic genes. After MCF7-V and MCF7-CT45A1 cells were cultured in serum-free medium (SF-EFB) for 5 days, expression of EMT, stemness, and metastatic genes in the cells was determined using semi-quantative RT-PCR (a). Expression levels of TWIST1 (b), ALDH1A1 (c), KIT (d), SULF2 (e), and CXCR4 (f) were quantitatively measured using QT-PCR. The experiments were repeated for three times. Samples were compared using one-way ANOVA, and error bars of the data indicated mean \pm S.D., ${ }^{* *} P<0.01$, as compared with MCF7-V cells 
a
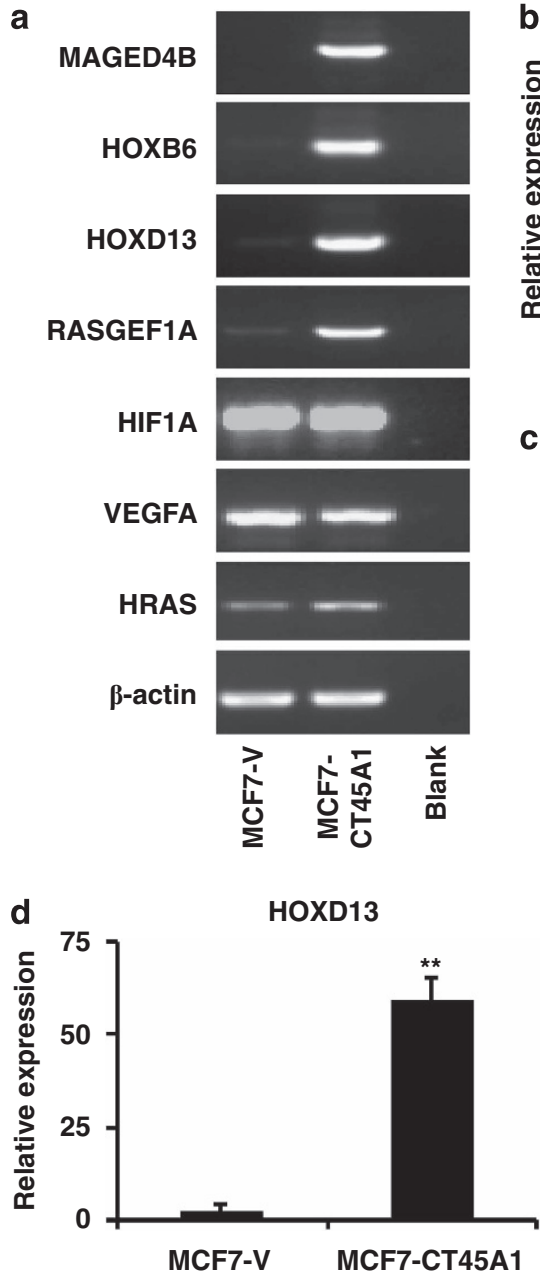

c
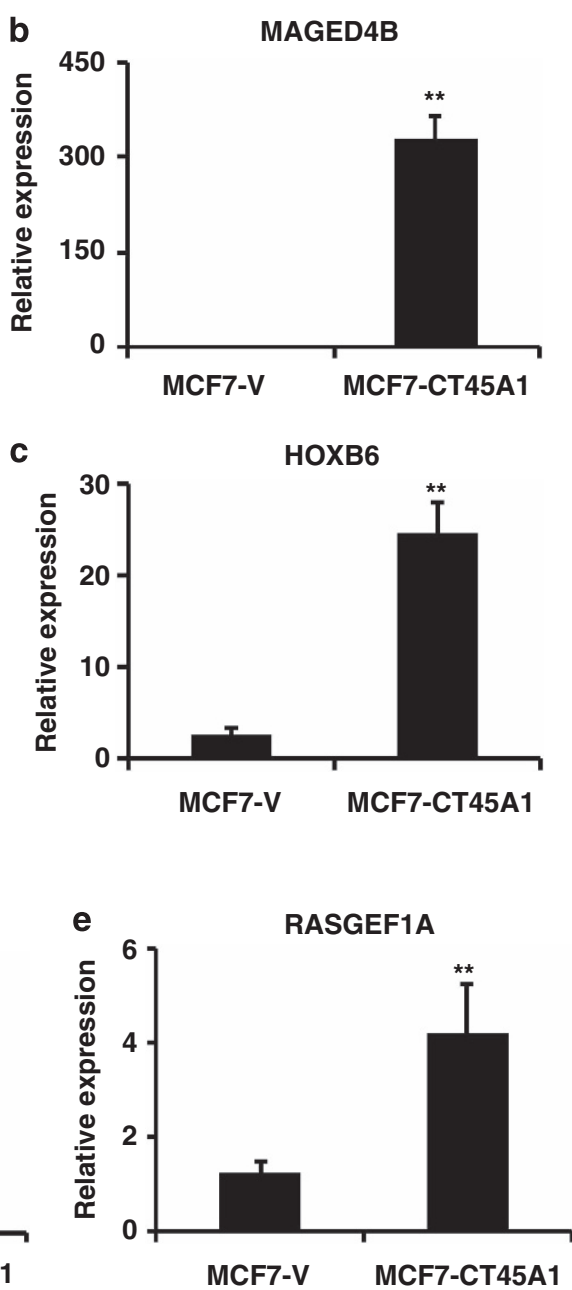

Figure 6 CT45A1 promotes the overexpression of tumorigenic genes. The expression of tumorigenic genes, including MAGED4B, HOXB6, HOXD13, RASGEF1A, HIF1A, VEGFA, and HRAS in MCF7-V and MCF7-CT45A1 cells was detected using RT-PCR (a); the expression levels of MAGED4B, HOXB6, HOXD13, and RASGEF1A were also measured using QT-PCR (b-e). The experiments were repeated for three times. Samples were compared using one-way ANOVA, and error bars of the data displayed mean \pm S.D., ${ }^{\star}{ }^{*} P<0.01$, as compared with MCF7-V cells

\section{Discussion}

During oncogenesis, tumors usually undergo an atavism that results in the overexpression of various embryonic development genes, including a variety of proto-oncogenes and CTAs. ${ }^{2-15}$ Among the more than 200 CTAs identified so far, CT45 is dynamically expressed during embryonic development and is silenced after birth but is reactivated in various cancer types in a favored tumor microenvironment; overexpression of CT45 is closely associated with poor overall survival of cancer patients. ${ }^{16-23}$ However, whether CT45 acts as proto-oncogenes has not been determined. ${ }^{2,3}$ In the current study, we found that overexpression of CT45A1 activated transcription of multiple oncogenic and metastatic genes, promoted EMT, increased breast cancer cell stemness and invasion, and increased metastasis in a growth factor-dependent manner. We therefore propose that CT45A1 acts as a new proto-oncogene, which activates the expression of numerous oncogenic genes, activating several key signaling pathways, and triggering tumorigenesis and cancer metastasis in a favored environment (Figure 8).
Overexpression of CT45A1 in breast cancer cells selectively increased the levels of several important EMT and stemness genes, including TWIST1, ALDH1A1, and KIT. Among them, TWIST1 is one of the dominant EMT genes overexpressed in various cancer types and promotes cancer cell EMT, migration, invasion, and metastasis ${ }^{26}$ ALDH1A1 is a well-known cancer stem cell marker. High ALDH1A1 activity has been found in a fast growing cell subpopulation with strong tumorigenicity. ${ }^{27,28} \mathrm{KIT}$ is a stem cell factor receptor and functions as a proto-oncogene in a variety of malignant tumors $^{29}$ and enhances stem/progenitor cell survival. ${ }^{30}$ Therefore, CT45A1-mediated overexpression of TWIST1, ALDH1A1, and KIT increases breast cancer cell EMT, dedifferentiation, and stemness.

In addition, CT45A1 promoted expression of four pro-oncogenic genes MAGED4B, HOXB6, HOXD13, and RASGEF1A. MAGED4B was originally identified in melanomas and is generally considered a tumor-specific antigen. Overexpression of MAGED4B promotes cell proliferation and EMT and nurtures tumorigenesis. ${ }^{31} \mathrm{HOX}$ genes are a highly conserved family of homeodomain-containing 
a

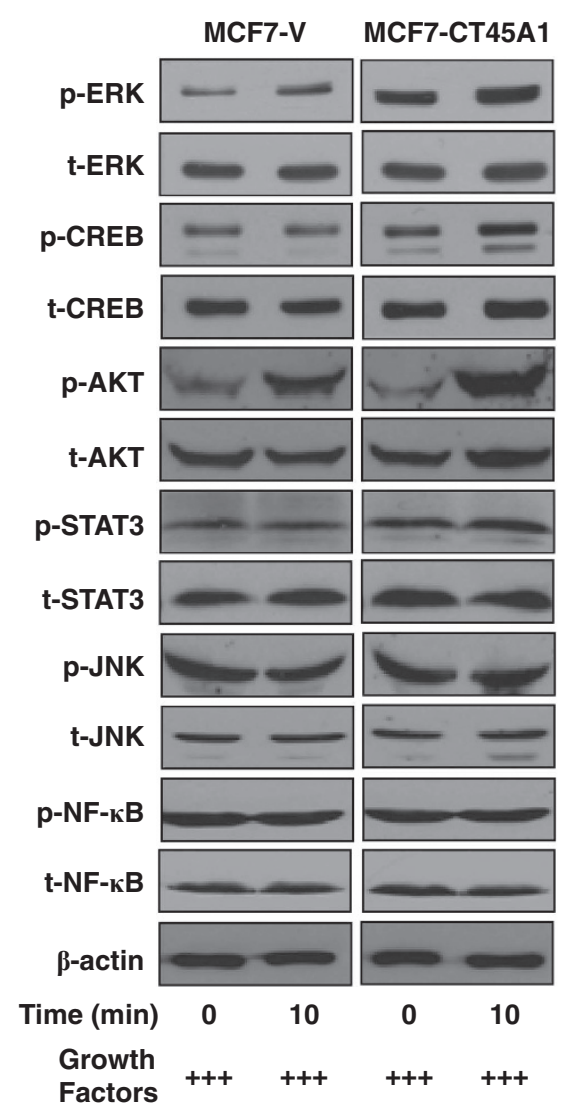

MW

(KD)

$42 / 44$

43

60

92

$46 / 54$

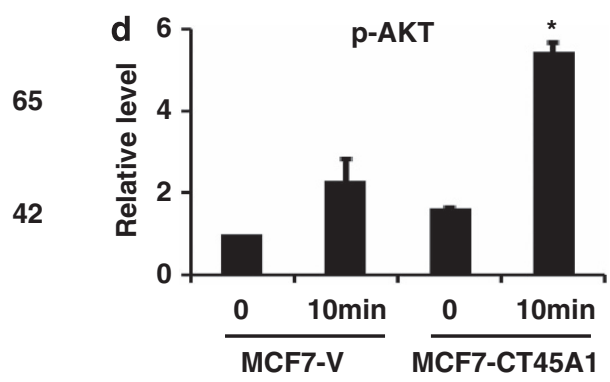

e

CT45A1

p-ERK
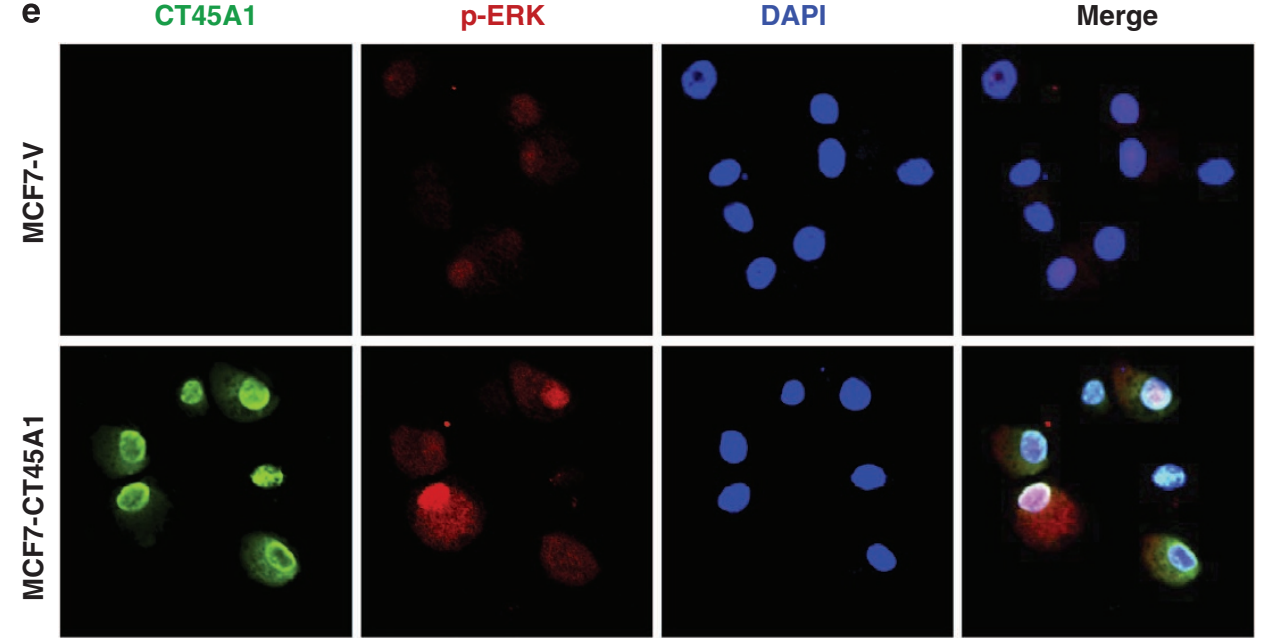

Figure 7 CT45A1 constitutively activates ERK and CREB signaling pathways. MCF7-V and MCF7-CT45A1 cells were activated with EGF, bFGF, and B27 at $37^{\circ} \mathrm{C}$ for $10 \mathrm{~min}$, and the total and phosphorylated proteins were detected using western blot analysis (a); the bands of p-ERK (b), p-CREB (c), and P-AKT (d) were scanned with grayscale scanning, and the density of the bands was statistically analyzed. The data were representative of two experiments. Samples were compared using one-way ANOVA, and error bars of the data displayed mean $\pm S . D$. ${ }^{*} P<0.05,{ }^{*} P<0.01$, as compared with MCF7-V cells. (e) MCF7-V and MCF7-CT45A1 cells on the slides were triply stained with CT45A1 antibody (green color), phosphorylated ERK antibody (red color), and DAPI (blue color), and the slides were photographed using confocal microscopy at $\times 1000$. The experiments were repeated twice

transcription factors. Accumulated data have shown that many silenced HOX genes in the normal body are reactivated in various malignant tumors. ${ }^{32}$ Among the HOX genes, HOXB6 is expressed in breast cancer cells. ${ }^{33}$ HOXD13 is widely overexpressed in a variety of cancer types and contributes to tumorigenesis. ${ }^{34}$ RASGEF1A has Ras guanyl nucleotide exchange factor activity and has an important role in RAS signaling. Its overexpression has been 


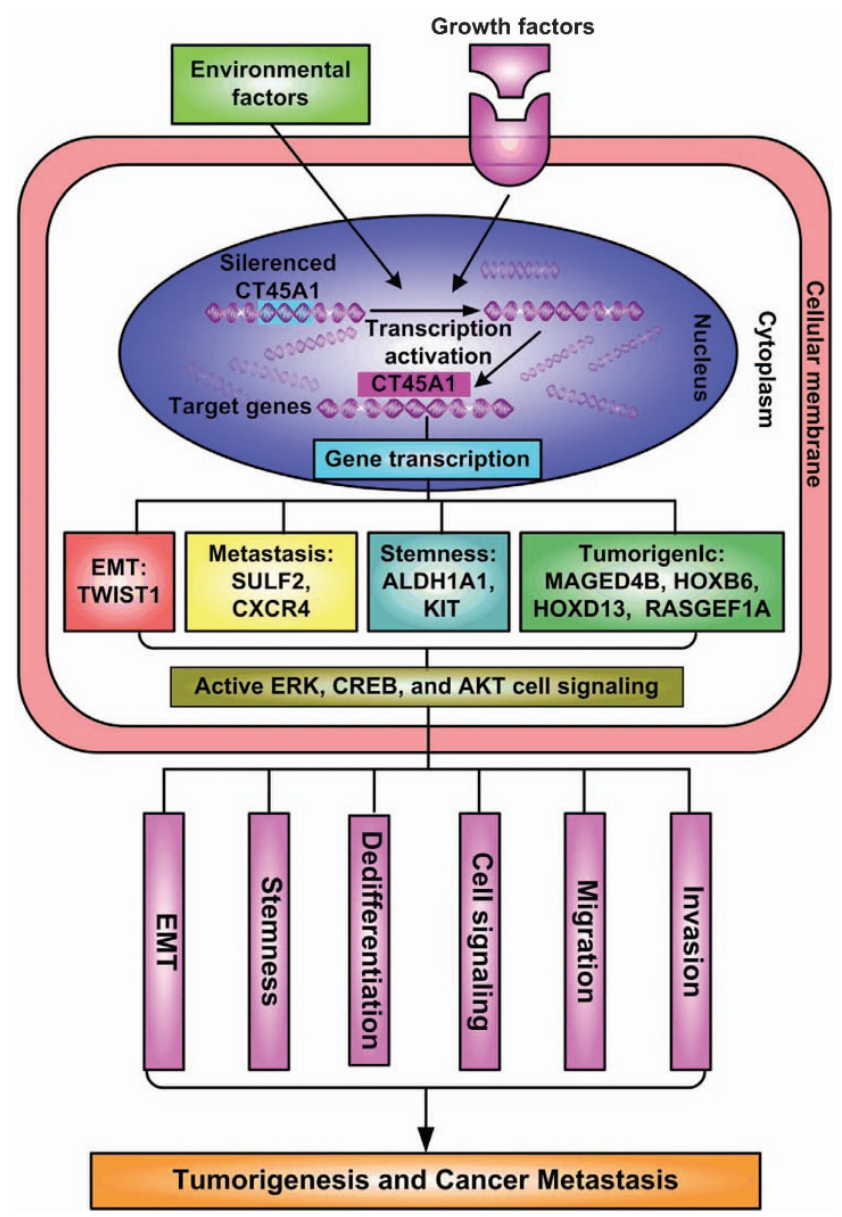

Figure 8 Schematic model for CT45A1 acts as a proto-oncogene to trigger tumorigenesis and cancer metastasis. Environmental factors and growth factors switch on transcription of the silenced CT45A1 gene in the nucleus. CT45A1 can directly bind to the promoter region of its target genes or interact with transcription factors to induce the transcription of several key tumorigenic and metastatic genes, including EMT master gene TWIST1, metastasis genes SULF2, and CXCR4, stemness genes ALDH1A1 and KIT, and tumorigenic genes MAGED4B, HOXB6, HOXD13, and RASGEF1A. In addition, overexpression of CT45 activates ERK, CREB, and AKT signaling pathways, induce breast cancer cell EMT and dedifferentiation, increase tumor cell stemness, migration, and invasion, and foster tumorigenesis and cancer metastasis

implicated in tumorigenesis. ${ }^{35,36}$ Hence, CT45A1-mediated overexpression of these pro-oncogenic genes may be major factors that trigger tumorigenesis in breast cancer.

Furthermore, overexpression of CT45A1 in breast cancer cells constitutively activated ERK and CREB signaling pathways in the absence of growth factor stimulation. It is well known that the constitutive activation of the RAS-MAPKERK signaling pathway drives uncontrolled cell proliferation, carcinogenesis, and cancer metastasis, ${ }^{35,36}$ and that CREB is overexpressed or over-activated in various cancer types and functions as a proto-oncogene to promote tumorigenesis and metastasis. ${ }^{37}$ Hence, CT45A1-mediated activation of ERK and CREB signaling pathways contribute to oncogenesis.

Moreover, CT45A1 expression dramatically elevated SULF2 expression levels. SULF2 is an endosulfatase that has a critical role in the regulation of cell migration and functions as a bona fide cancer-causing protein that promotes cancer metastasis. ${ }^{38-40}$ Thus, CT45A1-mediated overexpression of SULF2 may promote breast cancer cell invasion and metastasis. In addition, our data showed that CT45A1 induced CXCR4 overexpression in MCF7-CT45A1 cells and resulted in metastases from in situ breast tissue to lung tissue or to the vicinity of bone tissue. CXCR4 is involved in the activation of a key chemotaxis pathway and contributed to tumor cell invasion and metastasis. ${ }^{41-46}$ Thus, CT45A1induced high CXCR4 levels may cause breast cancer invasion and metastasis.

The mechanisms of CT45A1-mediated oncogenesis and cancer metastasis have not been determined, and several mechanistic questions need to be addressed. First, how do environmental factors activate the expression of silenced CT45A1 in cancer tissues? Second, among the CT45A1upregulated genes, which belong to its direct target genes, which are the secondarily affected genes? Third, does nuclear CT45A1 directly bind to promoters of its target genes or interact with transcription factors in the nucleus to stimulate gene transcription? Fourth, how does CT45A1 in collaboration with growth factors boost oncogenesis and cancer metastasis?

Analysis of putative domains in CT45A1 using InterProScan (http://www.ebi.ac.uk/interpro/) showed that the CT45A1 protein has a DEAD/H box that has a putative nucleic acidbinding function and RNA helicase activity (Supplementary Figure S1b). RNA helicase interacts with the C-terminus of RNA polymerase II (Pol II) to regulate gene transcription. However, whether CT45A1 interacts with Pol II and other transcription factors to drive the overexpression of various oncogenic and metastatic genes needs to be determined. Besides, our data showed that CT45A1 is mostly localized in the nucleus (Figure 7, Supplementary Figures S2d and S8c); the binding of the protein to its target genes and CT45A1mediated transcriptional regulatory mechanisms need to be further elucidated.

Interestingly, the CT45 gene family has a DEAD domain that is shared by the DEAD box proteins DDX26B, INTS6, and SAGE1. CT45 family members exist only in Homo sapiens and primates, ${ }^{15}$ belonging to novel genes in terms of biological evolution. Whether CT45 genes are derived from the three DEAD box proteins needs to be further studied. Hence, the CT45 family is a rare, but valuable, set of genes in the study of molecular phylogenetic trees and the genesis of novel genes during biological evolution.

As the CT45A1 protein is absent in normal tissues after birth, except for the testis, but highly expressed in various cancer types, particularly highly expressed in cancer stem/ progenitor cells, but not in differentiated cells, ${ }^{47}$ it is an attractive biomarker for diagnosis and prognostic predication of cancer patients and may be utilized to develop novel approaches for vaccination against cancer stem/progenitor cells and could be used as a target for novel anticancer drug discovery.

Malignant tumors usually undergo an atavism that overexpresses various embryonic development genes, including a variety of CTAs. Similar to many other CTAs, CT45 is dynamically expressed in a favored tumor microenvironment. Hence, CT45 is a good model system to study the dynamic activation of proto-oncogenes in tumor cells as a function of 
environment. In addition, CT45 and CTAs may be used as a tool to study the cross interactions between embryology and tumor biology. Therefore, further study of the oncogenic effects and mechanisms of CT45A1 and other CT45 family members as well as more than 200 CTAs should reveal new insights into tumorigenesis and provide new strategies and approaches for cancer diagnosis and specific tumor therapy.

\section{Materials and Methods}

Preparation of CT45A1 antigen and antibodies. CT45A1 CDNA was cloned into the pET-28a vector. The BL21-competent cells were transformed by CT45A1-pET-28a to produce recombinant CT45A1 protein. The recombinant CT45A1 protein was purified with Ni-NTA agarose, emulsified with complete Freund's adjuvant, and used in the primary and boosting immunization of seven $\mathrm{BALB} / \mathrm{c}$ mice, at doses of $100 \mu \mathrm{g} /$ mouse, and four rabbits, $500 \mu \mathrm{g} / \mathrm{rabbit}$, respectively. The blood of the immunized mice and rabbits were collected, and the specificity of these antibodies against CT45A1 was confirmed using WB and immunofluorescence (IF) staining.

Cell culture. The human breast cancer cell lines MCF7 and Hs578T, and human gastric cancer cell lines AGS and SGC7901were from ATCC. The cells were cultured in DMEM (high glucose) supplemented with $10 \%$ fetal bovine serum, $100 \mathrm{U} / \mathrm{ml}$ Penicillin $\mathrm{G}$, and $100 \mathrm{U} / \mathrm{ml}$ streptomycin (complete medium) under a humidified atmosphere of $5 \% \mathrm{CO}_{2}$ at $37^{\circ} \mathrm{C}$.

Infection of MCF7 cells. CT45A1 CDNA was cloned into the lentivirus vector Venus-IRES-GFP. The 293T cells were transfected with either CT45A1Venus-IRES-GFP or the control Venus-IRES-GFP to produce infectious viruses. MCF7 cells were infected with the lentivirus containing either CT45A1 cDNA or GFP CDNA, and the GFP-positive cells were sorted using flow cytometry, and the sorted MCF7 cells were named as MCF7-CT45A1 and MCF7-V, respectively. The expression of CT45A1 in the cells was confirmed using RT-PCR, WB, and IF (Supplementary Figure S2).

CT45A1 silence by shRNA. Four CT45A1 shRNAs, including shRNA19747 (5'-TGCAGTCAGGAAGCGATTT-3'), shRNA19748 (5'-GAGAAATTAATGCTGA TAT- $3^{\prime}$ ), shRNA19749 (5'-GATCCTGAAACTGTGTTA- ${ }^{\prime}$ ), and shRNA1 (5'-GGAG AGAAAAGGATCAGATT-3' $)^{24}$ that were cloned into the lentivirus vector GV248-GFP, were purchased from Shanghai Genechem Co., Ltd. (Shanghai, China). The infectious lentivirus was prepared by transfection of 293T cells with the CT45A1 shRNA constructs and control shRNA. SGC7901 cells were infected with five lentiviruses, respectively, and GFP-positive infected cells were sorted using flow cytometry. CT45A1 expression levels in the cells were determined using WB and IF staining.

Cell growth assay. Cell growth was measured with the AlamarBlue assay kit (Invitrogen, Carlsbad, CA, USA). Briefly, 3000 MCF7, Hs578T, AGS, and SGC7901 cells were seeded into each well of a 96-well plate, respectively. After culture for $46 \mathrm{~h}, 10 \mu \mathrm{l}$ AlamarBlue solution was added into each well and the plate was incubated at $37^{\circ} \mathrm{C}, 5 \% \mathrm{CO}_{2}$ for $2 \mathrm{~h}$, and the $\mathrm{OD}$ value was measured using a SpectraMax M5 multi-detection reader (Molecular Devices Corporation, Sunnyvale, CA, USA) at absorbance 560 and $590 \mathrm{~nm}$.

Cell cycle assay. MCF7, Hs578T, AGS, and SGC7901 cells were cultured in the complete medium for 2 days and the cells were fixed with $70 \%$ ethanol overnight at $4{ }^{\circ} \mathrm{C}$ and resuspended in $500 \mu \mathrm{l}$ PBS containing $100 \mathrm{mg} / \mathrm{l} \mathrm{RNasel}$. After incubation at $37^{\circ} \mathrm{C}$ for $30 \mathrm{~min}, 2.5 \mu \mathrm{l}(10 \mathrm{mg} / \mathrm{ml})$ propidium iodide (PI) solution was added. The DNA content of PI-stained cells was analyzed using flow cytometry (Becton Dickinson FACSCalibur, BD Biosciences, San Diego, CA, USA).

Cell apoptosis assay. MCF7 cells were seeded into each well of a six-well plate. The cells were irradiated with UV at $20 \mu \mathrm{J}$, cultured for $24 \mathrm{~h}$. The cells were stained with $\mathrm{Pl}$ and analyzed using flow cytometry. Besides, the apoptotic cells were also assessed with TUNEL assay using the ApopTag Red in Situ Apoptosis Detection Kit S7165 (Millipore, Shanghai, China) according to the manufacturer's instructions.

Mammosphere formation assay. MCF7 cells were first cultured for 5 days in either the complete medium or serum-free DMEM/F12 medium supplemented with $10 \mathrm{ng} / \mathrm{ml}$ EGF, $10 \mathrm{ng} / \mathrm{ml} \mathrm{bFGF}$, and $1 \times$ B27 (SF-EFB) under a humidified atmosphere of $5 \% \mathrm{CO}_{2}$ at $37^{\circ} \mathrm{C}$. Then, the cells were directly imaged with Intelligent Bio-image navigation FSX100 (Olympus Optical Co., Ltd, Tokyo, Japan). A sphere with more than 50 mammary cells is considered as a mammosphere.

Colony formation. One thousand MCF7 cells were cultured for 5 days in either the complete medium or SF-EFB medium, suspended in $100 \mu$ l of the complete medium, mixed with $150 \mu \mathrm{l}$ soft agarose and $1.5 \mathrm{ml}$ of the complete medium, and seeded into a $30-\mathrm{mm}$ plate overlying $1000 \mu \mathrm{l} 0.5 \%$ soft agar. The cells were cultured under a humidified atmosphere of $5 \% \mathrm{CO}_{2}$ at $37^{\circ} \mathrm{C}$ for 2 weeks and imaged with OLYMPUSSZX16 Research Stereo microscopy (Olympus Optical Co., Ltd). In addition, the colonies containing more than 50 cells were counted, and the data were analyzed with a SPSS 16.0 software (Statistical Product and Service solutions, Palo Alto, CA, USA).

Cell Wright-Giemsa staining. MCF7, Hs578T, AGS, and SGC7901 cells were transferred into each well of a six-well plate fitted with cover slips and cultured in the complete medium at $37^{\circ} \mathrm{C}$ with $5 \% \mathrm{CO}_{2}$ for $24 \mathrm{~h}$. The slides of MCF7 cells were prepared by a cytospin method and stained with Wright-Giemsa solution (Nanjing Jiancheng Bioengineering Institute, Nanjing, China) and photographed.

Cell migration assay. MCF7, Hs578T, AGS, and SGC7901 cells were seeded into each well of a six-well plate and incubated for $24 \mathrm{~h}$ to research near confluence. The cells were wounded with a plastic tip and cultured with RPMI1640 medium supplemented with $2 \%$ FBS at $37^{\circ} \mathrm{C}, 5 \% \mathrm{CO}_{2}$ for $48 \mathrm{~h}$. After Wright-Giemsa staining, the gaps and cells were imaged.

Cell invasion assay. MCF7, Hs578T, AGS, and SGC7901 cells were resuspended in serum-free medium at $5 \times 105 \mathrm{cells} / \mathrm{ml}$, and $100 \mu \mathrm{l}$ cells were seeded into the upper chamber (Corning Costar, Tewksbury, MA, USA) in serumfree medium. After incubation for $24 \mathrm{~h}$ at $37^{\circ} \mathrm{C}, 5 \% \mathrm{CO}_{2}$, the invaded cells on the lower chamber were fixed with $100 \%$ methanol, stained with Wright-Giemsa solution (Nanjing Jiancheng Bioengineering Institute) and photographed. Six randomly chosen fields were analyzed for each transwell.

Breast cancer in situ xenograft model. MCF7-V and MCF7-CT45A1 cells were cultured for 5 days in SF-EFB under a humidified atmosphere of $5 \% \mathrm{CO}_{2}$ at $37^{\circ} \mathrm{C}$. The $5 \times 10^{6} \mathrm{MCF} 7-\mathrm{V}$ or MCF7-CT45A1 cells were first mixed with $100 \mu \mathrm{l}$ of ice-cold Matrigel and then injected into the breast cushion of 8-week-old NOD-SCID mice ( $n=6$ each group). All mice used in this study were maintained in a laminar airflow cabinet under specific pathogen-free conditions in a 12-h light-dark cycle. Animal care and experiments followed approved animal protocols of the Soochow University Animal Care and Use Committee. The tumor sizes were measured and the mouse weight was weighted every other day. Tumor volume was calculated using the formula: length $\times$ width $^{2} \times 0.55$. After 40 days, the tumor invasion in the breast area of the mice was photographed, and the tumors were excised and weighted, and the lung, liver, spleen, and kidney tissues were also collected.

H\&E staining. In brief, the tumors and tissues were fixed and sectioned. After dewaxing rehydration, the slides were prepared and stained with H\&E staining solution. The slides were mounted and observed using OLYMPUS microscopy.

Cell and tissue immunofluorescent staining. Cells were fixed in $4 \%$ paraformaldehyde for $10 \mathrm{~min}$ at room temperature and permeated with $0.1 \%$ Triton X-100 for $15 \mathrm{~min}$. The tumors and tissues of in situ xenografted mice were fixed, and sectioned, and dewaxing rehydration was performed. The slides were prepared and blocked with $5 \% \mathrm{BSA}$ and incubated overnight at $4{ }^{\circ} \mathrm{C}$ with antibodies against CT45A1, CD44, and phosphorylated ERK, followed by incubation with Alexa Fluor 594 and/or 488-coupled secondary antibodies for $1 \mathrm{~h}$ at room temperature. The cell nuclei were stained with 4.6-diamidino-2phenylindole (DAPI) for $10 \mathrm{~min}$. The slides were mounted and imaged using OLYMPUS confocal microscopy (Olympus Optical Co., Ltd).

DNA microarray. The total RNA in the MCF7 cells was purified with the Trizol kit (Invitrogen), and the differential gene expression between MCF7-V and MCF7-CT45A1 cells was analyzed using the U133 Plus 2.0 Array 
(Affymetrix, Santa Clara, CA, USA, containing 48000 transcripts, total 32375 human genes) by the Shanghai Biotechnology Co., Ltd. (Shanghai, China).

RT-PCR and QT-PCR. The total RNA was extracted from MCF7-V or MCF7CT45A1 cells, and the CDNA was generated by reverse transcription using the RevertAid First Strand cDNA Synthesis Kit (Fermentas Life Sciences, Chicago, NY, USA). RT-PCR was performed as we previously described and PCR primers were listed in Supplementary Table 3. PCR products were resolved on $1.5 \%$ agarose gel and the bands were imaged. QT-PCR was carried out by Applied Biosystems ABI 7500 (ABI, Carlsbad, CA, USA) and using SYBR Green PCR Master Mix (ABI) and primers listed in Supplementary Table 4. Briefly, the PCR reaction consisted of an initial denaturation at $95^{\circ} \mathrm{C}$ for $10 \mathrm{~min}$, followed by denaturation at $94^{\circ} \mathrm{C}$ for $15 \mathrm{~s}$, and annealing and extension at $60^{\circ} \mathrm{C}$ for $1 \mathrm{~min}$ for each PCR cycle. After a total of 40 cycles, the QT-PCR results were analyzed with a QT-PCR-data processing software of the machine.

Western blotting. The proteins in MCF7, Hs578T, AGS, and SGC7901 cells were extracted with the M-PER Mammalian Protein Extraction Kit (Thermo Fisher Scientific, Waltham, MA, USA). Equal amounts of the proteins were loaded on each lane and resolved using SDS-PAGE with Tris-glycine running buffer for $50 \mathrm{~min}$ at $150 \mathrm{~V}$. The proteins were transferred to nitrocellulose membranes by wet electrotransfer for $90 \mathrm{~min}$ at $250 \mathrm{~mA}$. The membranes were blocked with $5 \%$ nonfat milk and incubated overnight at $4{ }^{\circ} \mathrm{C}$ with primary antibodies against CT45A1 or other proteins, followed by incubation with HRP-coupled secondary antibody for $1 \mathrm{~h}$. The blots were visualized using enhanced chemiluminescence detection reagents and exposed to X-ray film. The blots were then stripped and reprobed with the HRP-labeled anti- $\beta$-actin antibody (Sigma, St. Louis, MO, USA).

Cell signaling. MCF7 cells were suspended in PBS at a density of $5.0 \times$ $10^{7} \mathrm{cell} / \mathrm{s} / \mathrm{ml}$. Cells $(100 \mu \mathrm{l})$ were incubated with EGF $(50 \mathrm{ng} / \mathrm{ml}), \mathrm{bFGF}(50 \mathrm{ng} / \mathrm{ml})$, and B27 $(5 \times)$ at $37^{\circ} \mathrm{C}$ for $10 \mathrm{~min}$, and then stopped with $100 \mu \mathrm{l} 2 \times$ SDS sample buffer with $10 \mathrm{mM}$ sodium vanadate, $10 \mathrm{mM}$ sodium fluoride, $10 \mathrm{mM}$ DTT, and denaturated at $95^{\circ} \mathrm{C}$ for $3 \mathrm{~min}$. The total and phosphorylated ERK, CREB, AKT, JNK, NF- $\kappa$ B (Cell Signaling Technology, Boston, MA, USA), and STAT3 (BD Pharmingen, San Diego, CA, USA) were detected using WB analysis.

Statistical analysis. All data in this study were expressed as mean \pm S.D. or S.E.M. and statistically compared using one-way ANOVA. $P<0.05$ is considered as statistically significant, and $P<0.01$ as very significant.

\section{Conflict of Interest}

The authors declare no conflict of interest.

Acknowledgements. This study was supported by grants from the National Natural Science Foundation of China (Grant no. 81372376), Suzhou City Scientific Research Funds (nos. SS201004 and SS201138), a project funded by the priority academic program development of the Jiangsu Higher Education Institutions (PAPD), Cultivation base of the State Key Laboratory of Stem Cell and Biomaterials built together by the Ministry of Science and Technology and Jiangsu Province, Jiangsu Province's Key Discipline of Medicine (XK201118), Undergraduate Creative Business Training Plan (nos. 5731554512 and SG31554512), and 2011 Collaborative Innovation Center of Hematology, Jiangsu Province.

1. Catalano V, Turdo A, Di Franco S, Dieli F, Todaro M, Stassi G et al. Tumor and its microenvironment: a synergistic interplay. Semin Cancer Biol 2013; 23: 522-532.

2. Fratta E, Coral S, Covre A, Parisi G, Colizzi F, Danielli R et al. The biology of cancer testis antigens: putative function, regulation and therapeutic potential. Mol Oncol2011; 5: 164-182.

3. Whitehurst AW. Cause and consequence of cancer/testis antigen activation in cancer. Annu Rev Pharmacol Toxicol 2013; 54: 251-272.

4. der Bruggen Pv, Traversari C, Chomez P, Lurquin C, De Plaen E, Van den Eynde B et al. A gene encoding an antigen recognized by cytolytic $T$ lymphocytes on a human melanoma. Science 1991; 254: 1643-1647.

5. Traversari C, van der Bruggen P, Luescher IF, Lurquin C, Chomez P, Van Pel A et al. A nonapeptide encoded by human gene MAGE-1 is recognized on HLA-A1 by cytolytic T lymphocytes directed against tumor antigen MZ2-E. J Exp Med 1992; 176: 1453-1457.

6. Chen YT, Boyer AD, Viars CS, Tsang S, Old LJ, Arden KC et al. Genomic cloning and localization of CTAG, a gene encoding an autoimmunogenic cancer-testis antigen NY-ESO-1, to human chromosome Xq28. Cytogenet Cell Genet 1997; 79: 237-240.
7. John T, Starmans MH, Chen YT, Russell PA, Barnett SA, White SC et al. The role of Cancer-Testis antigens as predictive and prognostic markers in non-small cell lung cancer. PLoS One 2013; 8: e67876.

8. Ogata K, Aihara R, Mochiki E, Ogawa A, Yanai M, Toyomasu Y et al. Clinical significance of melanoma antigen-encoding gene-1 (MAGE-1) expression and its correlation with poor prognosis in differentiated advanced gastric cancer. Ann Surg Oncol 2011; 18: 1195-1203.

9. Chen Z, Li M, Yuan Y, Wang Q, Yan L, Gu J et al. Cancer/testis antigens and clinical risk factors for liver metastasis of colorectal cancer: a predictive panel. Dis Colon Rectum 2010; 53: 31-38.

10. Pandey A, Kurup A, Shrivastava A, Radhi S, Nguyen DD, Arentz C et al. Cancer testes antigens in breast cancer: biological role, regulation, and therapeutic applicability. Int Rev Immunol 2012; 31: 302-320.

11. Shiraishi T, Terada N, Zeng Y, Suyama T, Luo J, Trock B et al. Cancer/testis antigens as potential predictors of biochemical recurrence of prostate cancer following radical prostatectomy. J Transl Med 2011; 9: 153.

12. von BL, Keller L, Mortezavi A, Provenzano M, Sais G, Hermanns T et al. MAGE-C2/CT10 protein expression is an independent predictor of recurrence in prostate cancer. PLoS One 2011; 6: e21366.

13. Ayyoub M, Taub RN, Keohan ML, Hesdorffer M, Metthez G, Memeo L et al. The frequent expression of cancer/testis antigens provides opportunities for immunotherapeutic targeting of sarcoma. Cancer Immun 2004; 4: 7.

14. Inaoka RJ, Jungbluth AA, Gnjatic S, Ritter E, Hanson NC, Frosina D et al. Cancer/testis antigens expression and autologous serological response in a set of Brazilian nonHodgkin's lymphoma patients. Cancer Immunol Immunother 2012; 61: 2207-2214.

15. Dobrynin P, Matyunina E, Malov SV, Kozlov AP. The novelty of human cancer/testis antigen encoding genes in evolution. Int J Genomics 2013; 2013: 105108.

16. Chen YT, Hsu M, Lee P, Shin SJ, Mhawech-Fauceglia $P$ et al. Cancer/testis antigen CT45: analysis of mRNA and protein expression in human cancer. Int $J$ Cancer 2009; 124: 2893-2898.

17. Chen YT, Ross DS, Chiu R, Zhou XK, Chen YY et al. Multiple cancer/testis antigens are preferentially expressed in hormone-receptor negative and high-grade breast cancers. PLoS One 2011; 6: e17876.

18. Zhou $X$, Yang $F$, Zhang $T$, Zhuang $R$, Sun $Y$, Fang $L$ et al. Heterogeneous expression of CT10, CT45 and GAGE7 antigens and their prognostic significance in human breast carcinoma. Jpn J Clin Oncol 2013; 43: 243-250.

19. Andrade VC, Vettore AL, Regis Silva MR, Felix RS, Almeida MS, de Carvalho F et al. Frequency and prognostic relevance of cancer testis antigen 45 expression in multiple myeloma. Exp Hematol 2009; 37: 446-449.

20. Cerveira N, Meyer C, Santos J, Torres L, Lisboa S, Pinheiro M et al. A novel spliced fusion of MLL with CT45A2 in a pediatric biphenotypic acute leukemia. BMC Cancer 2010; 10: 518.

21. Heidebrecht HJ, Claviez A, Kruse ML, Pollmann M, Buck F, Harder S et al. Characterization and expression of CT45 in Hodgkin's lymphoma. Clin Cancer Res 2006; 12: 4804-4811.

22. Chen YT, Chadburn A, Lee P, Hsu M, Ritter E, Chiu A et al. Expression of cancer testis antigen CT45 in classical Hodgkin lymphoma and other B-cell lymphomas. Proc Natl Acad Sci USA 2010; 107: 3093-3098.

23. Inaoka RJ, Jungbluth $A A$, Baiocchi OC, Assis MC, Hanson NC, Frosina D et al. An overview of cancer/testis antigens expression in classical Hodgkin's lymphoma (cHL) identifies MAGE-A family and MAGE-C1 as the most frequently expressed antigens in a set of Brazilian CHL patients. BMC Cancer 2011; 11: 416.

24. Koop A, Sellami N, Adam-Klages S, Lettau M, Kabelitz D, Janssen $O$ et al. Down-regulation of the cancer/testis antigen 45 (CT45) is associated with altered tumor cell morphology, adhesion and migration. Cell Commun Signal 2013; 11: 41.

25. Nairismagi ML, Vislovukh A, Meng Q, Kratassiouk G, Beldiman C, Petretich $M$ et al. Translational control of TWIST1 expression in MCF-10A cell lines recapitulating breast cancer progression. Oncogene 2012; 31: 4960-4966.

26. Gajula RP, Chettiar ST, Williams RD, Thiyagarajan S, Kato Y, Aziz K et al. Required for Twist1-induced prostate cancer metastasis. Mol Cancer Res 2013; 11: 1387-1400.

27. Isfoss BL, Holmqvist B, Jernstrom H, Alm P, Olsson H. Women with familial risk for breast cancer have an increased frequency of aldehyde dehydrogenase expressing cells in breast ductules. BMC Clin Pathol 2013; 13: 28.

28. Charafe-Jauffret E, Ginestier C, Bertucci F, Cabaud O, Wicinski J, Finetti P et al. ALDH1-positive cancer stem cells predict engraftment of primary breast tumors and are governed by a common stem cell program. Cancer Res 2013; 73: 7290-7300.

29. Miettinen M, Lasota J. KIT (CD117): a review on expression in normal and neoplastic tissues, and mutations and their clinicopathologic correlation. Appl Immunohistochem Mol Morphol 2005; 13: 205-220.

30. Regan JL, Kendrick H, Magnay FA, Vafaizadeh V, Groner B, Smalley MJ. c-Kit is required for growth and survival of the cells of origin of Brca1-mutation-associated breast cancer. Oncogene 2012; 31: 869-883.

31. Germano S, Kennedy S, Rani S, Gleeson G, Clynes M, Doolan P et al. MAGE-D4B is a novel marker of poor prognosis and potential therapeutic target involved in breast cancer tumorigenesis. Int J Cancer 2012; 130: 1991-2002.

32. Shah N, Sukumar S. The Hox genes and their roles in oncogenesis. Nat Rev Cancer 2010; 10: $361-371$ 
33. Fischbach NA, Rozenfeld S, Shen W, Fong S, Chrobak D, Ginzinger D et al. HOXB6 overexpression in murine bone marrow immortalizes a myelomonocytic precursor in vitro and causes hematopoietic stem cell expansion and acute myeloid leukemia in vivo. Blood 2005; 105: 1456-1466.

34. Cantile M, Franco R, Tschan A, Baumhoer D, Zlobec I, Schiavo G et al. HOX D13 expression across 79 tumor tissue types. Int $J$ Cancer 2009; 125: 1532-1541.

35. Whelan JT, Hollis SE, Cha DS, Asch AS, Lee MH. Post-transcriptional regulation of the Ras-ERK/MAPK signaling pathway. J Cell Physiol 2012; 227: 1235-1241.

36. De Luca A, Maiello MR, D'Alessio A, Pergameno M, Normanno N. The RAS/RAF/MEK ERK and the PI3K/AKT signalling pathways: role in cancer pathogenesis and implications for therapeutic approaches. Expert Opin Ther Targets 2012; 16(Suppl 2): S17-S27.

37. Siu YT, Jin DY. CREB-a real culprit in oncogenesis. FEBS J 2007; 274: 3224-3232.

38. Morimoto-Tomita M, Uchimura K, Bistrup A, Lum DH, Egeblad M et al. Sulf-2, a proangiogenic heparan sulfate endosulfatase, is upregulated in breast cancer. Neoplasia 2005; 7: 1001-1010.

39. Gill RB, Day A, Barstow A, Liu H, Zaman G, Dhoot GK et al. Sulf2 gene is alternatively spliced in mammalian developing and tumour tissues with functional implications. Biochem Biophys Res Commun 2011; 414: 468-473.

40. Bret C, Moreaux J, Schved JF, Hose D, Klein B. SULFs in human neoplasia: implication as progression and prognosis factors. J Transl Med 2011; 9: 72.

41. Muller A, Homey B, Soto H, Ge N, Catron D, Buchanan ME et al. Involvement of chemokine receptors in breast cancer metastasis. Nature 2001; 410: 50-56.

42. Murphy PM. Chemokines and the molecular basis of cancer metastasis. $N$ Engl J Med 2001; 345: 833-835.
43. Furusato B, Mohamed A, Uhlen M, Rhim JS. CXCR4 and cancer. Pathol Int 2010; 60: 497-505.

44. Xu TP, Shen H, Liu LX, Shu YQ. The impact of chemokine receptor CXCR4 on breast cancer prognosis: a meta-analysis. Cancer Epidemiol 2013; 37: 725-731.

45. Burger JA, Kipps TJ. CXCR4: a key receptor in the crosstalk between tumor cells and their microenvironment. Blood 2006; 107: 1761-1767.

46. Mukherjee D, Zhao J. The Role of chemokine receptor CXCR4 in breast cancer metastasis. Am J Cancer Res 2013; 3: 46-57.

47. Yamada R, Takahashi A, Torigoe $T$, Morita R, Tamura $Y$, Tsukahara $T$ et al. Preferential expression of cancer/testis genes in cancer stem-like cells: proposal of a novel sub-category, cancer/testis/stem gene. Tissue Antigens 2013; 81: $428-434$.

Cell Death and Disease is an open-access journal published by Nature Publishing Group. This work is licensed under a Creative Commons Attribution-NonCommercialShareAlike 3.0 Unported License. The images or other third party material in this article are included in the article's Creative Commons license, unless indicated otherwise in the credit line; if the material is not included under the Creative Commons license, users will need to obtain permission from the license holder to reproduce the material. To view a copy of this license, visit http://creativecommons.org/ licenses/by-nc-sa/3.0/

Supplementary Information accompanies this paper on Cell Death and Disease website (http://www.nature.com/cddis) 